\title{
Controles litológicos y texturales de la mineralización tipo Mississippi Valley de los mantos en Shalipayco, Junín, Perú
}

\author{
Lithological and textural controls of Mississippi Valley type mineralization of \\ mantles in Shalipayco, Junín, Peru
}

\author{
Nancy Tuanama Tuanama ${ }^{1}$ Enrique Guadalupe Gómez ${ }^{2}$
}

Recibido: 05/08/2020 - Aprobado: 01/02/2021 - Publicado: 18/06/2021

\begin{abstract}
RESUMEN
La problemática a resolver era que tipos de controles litológicos, texturales y estructurales eran favorables a la mineralización del yacimiento Shalipayco y para lo cual, se realizó la exploración geoquímica, estudios sedimentológicos, cartografiado geológico, columnas estratigráficas y perforación diamantina, realizándose muestreos de superficie y de testigos de perforación y se encontro que los Mantos mineralizados del proyecto Shalipayco están ubicados litoestratigraficamente dentro de las rocas del Grupo Pucará, específicamente en la Formación Chambará y dentro de ella el control litológico de los mantos mineralizados son las dolomías de grano grueso a medio (wackestone y packestone) que presentan porosidad tipo vuggy y a las texturas más importantes favorables a la mineralización de los mantos son las brechas tectónicas evaporíticas, brechas evaporíticas, crackel brecha, pseudobrechas evaporíticas, y dolomías porosas donde las cavidades de disolución están rellenadas por dolomita cristalizada y/o sulfuros de $\mathrm{Zn}$ y $\mathrm{Pb}$, se ha reconocido también como control estructural de mineralización a fallas de alto ángulo, finalmente se ha encontrado los siguientes mantos mineralizados, Virgencita, Intermedio y Resurgidora con clara tipología de ser un yacimiento tipo Valle del Mississippi.
\end{abstract}

Palabras claves: Brecha; mineralizacion; control litológico.

\section{ABSTRACT}

The problem to be solved was which types of lithological, textural and structural controls were favorable to the mineralization of the Shalipayco deposit. Geochemical exploration, sedimentological studies, geological mapping, stratigraphic columns and diamond drilling were carried out, furthermore, performing surface and drill core sampling. It was found mineralized mantos of the Shalipayco project are located lithostratigraphically within the rocks of the Pucara Group, specifically in the Chambara Formation with lithological control of the mineralized mantos, they are coarse to medium grained dolomites (wackestone and packstone) present vuggy type porosity, and the most important textures favorable to the mineralization of the mantos are the evaporitic tectonic breccias, evaporitic breccias, crackel breccia, evaporitic pseudobreccias, and porous dolomites where the dissolution cavities are filled by crystallized dolomite and/or $\mathrm{Zn}$ and $\mathrm{Pb}$ sulfides. Recognized structural control of mineralization are high angle faults, finally, it has been found the following mineralized mantos, Virgencita, Intermedio and Resurgidora with clear typology of being a Mississippi Valley type deposit.

Keywords: Breccia; mineralization; lithological control.

1 Universidad Nacional Mayor de San Marcos, Facultad de Ingeniería Geológica, Minera, Metalúrgica y Geográfica, Unidad de Posgrado. Lima, Perú. E-mail: nancy.tuanama@gmail.com - ORCID: https://orcid.org/0000-0002-0281-269X

2 Universidad Nacional Mayor de San Marcos, Facultad de Ingeniería Geológica, Minera, Metalúrgica y Geográfica, Unidad de Posgrado. Lima, Perú. E-mail: eguadalupeg@unmsm.edu.pe - ORCID: https://orcid.org/0000-0001-9583-8807 


\section{INTRODUCCIÓN}

El proyecto Shalipayco es un depósito Tipo Mississippi Valley ubicado en la localidad de Shalipayco, distrito de Carhuamayo, provincia y departamento de Junín, a 15 kilómetros al noreste de la ciudad de Carhuamayo a una altitud variable entre los 4,200 y 4,800 m.s.n.m., (figura 1). En el año 2008, Votorantim Metais comenzó los trabajos de exploración (geoquímica, mapeo geológico, perforación diamantina e interpretaciones). Actualmente, el Proyecto Shalipayco se encuentra en etapa de factibilidad a cargo de Nexa Resources Peru en join venture con Pan American Silver Peru. El Proyecto cuenta con recursos medidos e indicados de $9.47 \mathrm{Mt} ; 4.44 \% \mathrm{Zn} ; 0.48 \% \mathrm{~Pb} ; 31.1 \mathrm{~g} / \mathrm{tAg}$. (MINEM, 2020).

La hipótesis en esta investigación se cumple ya que controles geológicos como los litológicos, estructurales y texturales de mineralización en el depósito Shalipayco están relacionados a la mineralización de tipo Mississippi Valley. El aporte principal es el estudio detallado de texturas que se relacionan con la mineralización.

\section{MÉTODOS}

El trabajo de investigación es descriptivo y explicativo se ha realizado principalmente con la técnica de la observación. Se realizaron estudios sedimentológicos y litoestratigráficos que fue complementado con investigaciones que determinan que la Formación Chambará tiene $622 \mathrm{~m}$. de potencia y se compone casi exclusivamente de dolomías intercaladas con pocas dolomías calcáreas, calizas dolomíticas, y calizas. Dentro de la Formación Chambará se presenta mineralización en mantos tipo Mississippi Valley principalmente en tres posiciones estratigráficas distintas; la primera directamente sobre el Grupo Mitú, la segunda aproximadamente entre los 140 y $200 \mathrm{~m}$. de la columna, y la tercera entre 460 y $480 \mathrm{~m}$. de la columna en bancos oolíticos y algal mats, en cristal de dolomita de tamaño muy grueso, asociada a esos mantos (Rosas \& Fontboté, 1989). El bitumen suele ocurrir con frecuencia asociado con la pirita en las zonas con cambios de facies, en el techo las estructuras falladas y brechas hidráulicas. En el año 2008, Votorantim Metais y Panamerican Silver generan un join venture y así Votorantim Metais comenzó los trabajos de exploración (geoquímica, cartografiado geológico, perforación diamantina e interpretaciones). Se cartografió a escala $1 / 10,000$, identificando las principales unidades estratigráficas, estructuras y zonas favorables de mineralización, luego se detalló a escala 1/2,000 para definir los cambios litológicos entre calizas y dolomías asi como los marcadores litológicos, ocurrencias de óxidos y/o sulfuros, una vez localizado un nivel mineralizado se describió y se procedió con el muestreo del mismo. Se elaboró columnas estratigráficas para diferenciar las facies litológicas y mineralógicas. Para realizar la selección de los diferentes datos se recolectó muestras de roca y mineral tanto de superficie como de los diferentes sondajes.

\section{RESULTADOS}

\subsection{Geología regional}

El area del Proyecto Shalipayco se distinguen por dos franjas, de rumbo NO-SE, bien definidas y separadas por un sobreescurrimiento (Falla Ulcumayo-San Rafael de rumbo NO-SE). En el sector oriental existen afloramientos de materiales pre-andinos con basamento, éstos son el complejo metamórfico e intrusivo de Huaytapallana (Precámbrico), y las areniscas y volcánicos del Grupo Mitú (Pérmico medio-Triásico Inferior). Las rocas del Grupo Pucará constituyen un monoclinal de rumbo NO-SE que buza hacia el sur. El sector occidental está limitado por el cabalgamiento del Grupo Mitú sobre el Grupo Pucará, se caracteriza por la presencia de un anticlinal NO-SE en cuyo núcleo se encuentran los sedimentos clásticos del Grupo

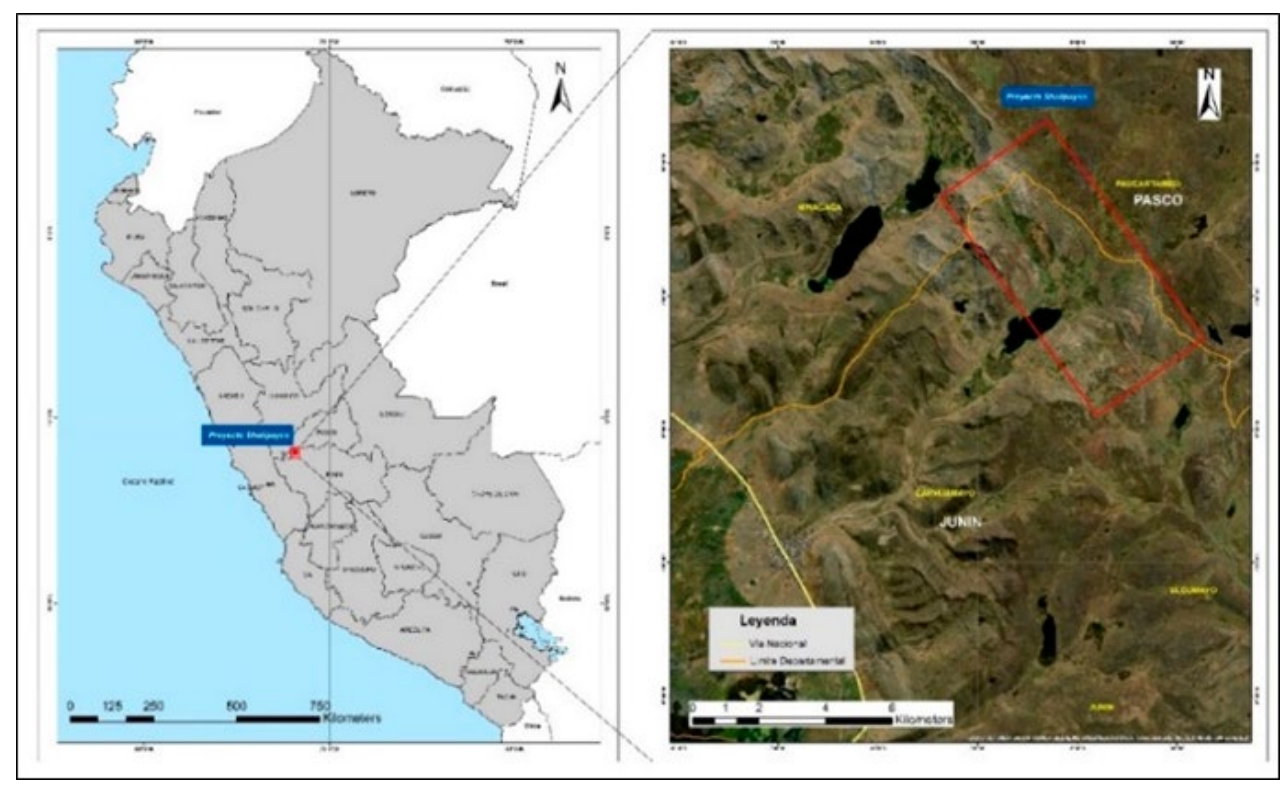

Figura 1. Ubicación del Proyecto Shalipayco 
Ambo (Mississipiano) y en los flancos los materiales del Grupo Mitú (MINEM, 2010).

\subsection{Geología local}

La geología del proyecto se caracteriza por afloramientos de rocas carbonatadas del Grupo Pucará (Formaciones Chambará, Aramachay y Condorsinga) que suprayacen a las rocas volcanoclásticas del Grupo Mitú (Pérmico); (figura 2). En la columna estratigráfica del área de estudio del proyecto Shalipayco se pueden observar las secuencias de la Formación Chambará y su relación con la mineralización (figura 3).

\subsection{Geología estructural}

Las estructuras predominantes presentan rumbo andino (NO-SE), mayormente fallas inversas, sobreescurrimientos y pliegues (figura 2). Se observan fallas N-S que son las más antiguas. Las fallas NE-SO con rumbos $\mathrm{N} 45^{\circ} \mathrm{E}$ y buzamiento $85^{\circ}$ al SE que constituye un evento posterior al sistema N-S, al que en algunos casos desplaza. Las fallas NNE-SSO ocurren entre las fallas mayores de los sistemas anteriores, con acomodamiento de bloques fallados $y$ fracturados.

\subsection{Mineralización}

La mineralización se encuentra principalmente en forma de mantos dentro de la Formación Chambará controlada por la litología y texturas con niveles de dolomita de tamaño de cristal medio a grueso y porosas. Las fallas de fuerte buzamiento son conocidas como principales conductores de la mineralización de los depósitos tipo Mississippi Valley, (Leach et al., 2010). Las principales ocurrencias de mineralización de tipo manto son:

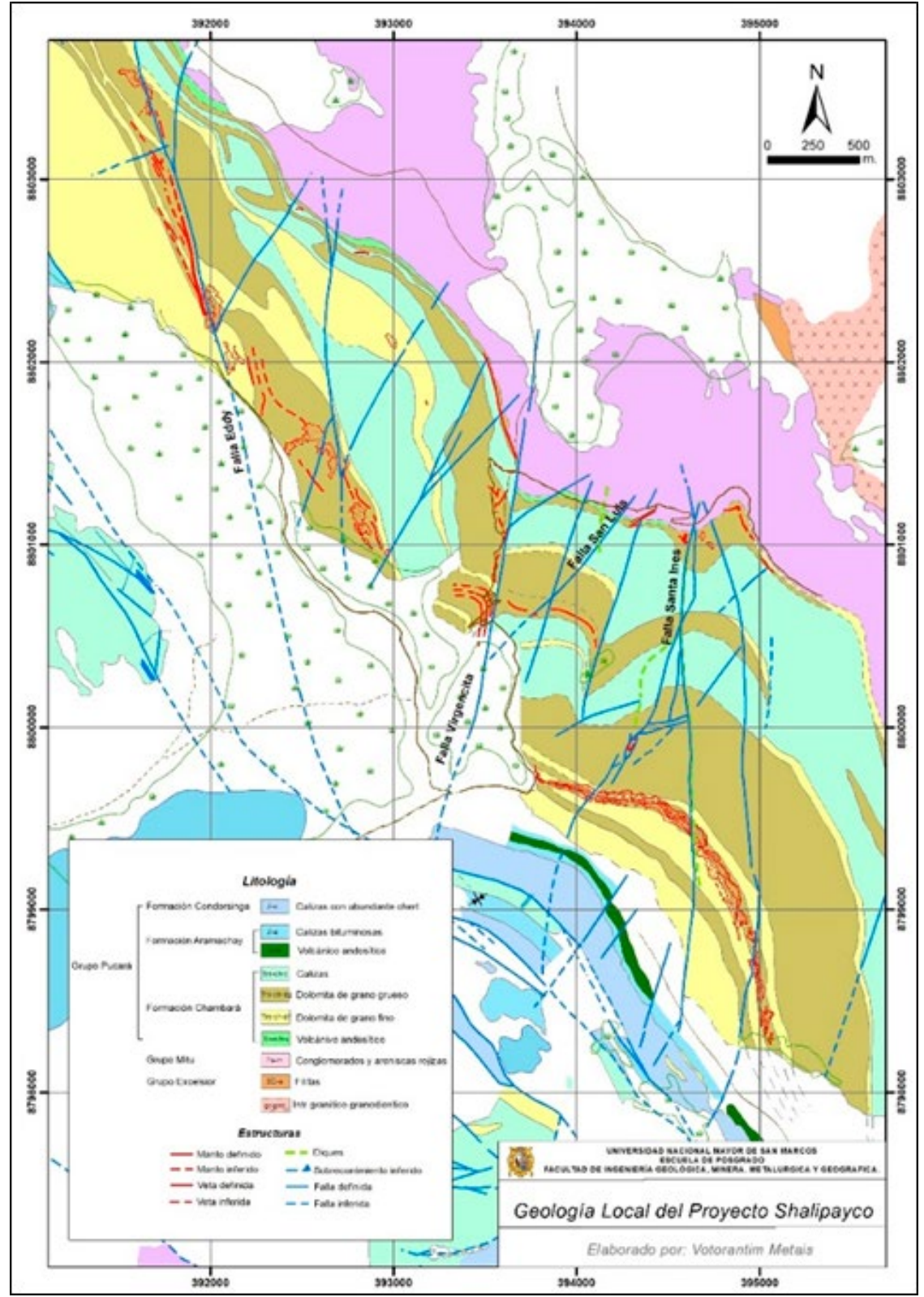

Figura 2. Mapa geológico local de la zona del proyecto, donde se observa los principales afloramientos del Grupo Mitú, Grupo Pucará y rocas intrusivas (Votorantim Metais, 2010) 


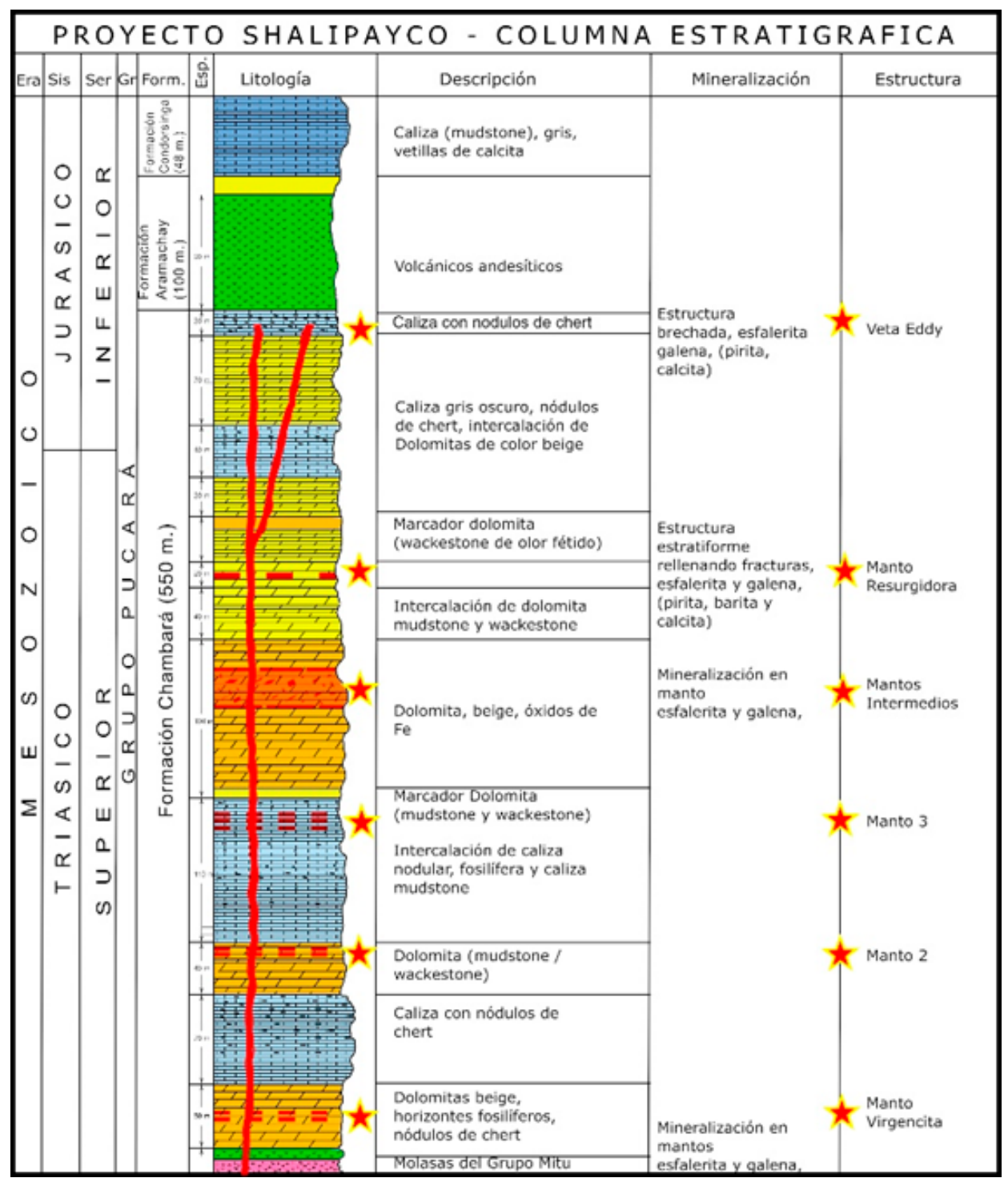

Figura 3. Columna estratigráfica del proyecto Shalipayco, donde se observa las posiciones estratigráficas de los principales mantos mineralizado (Votorantim Metais, 2010)

\subsubsection{Manto Virgencita (contacto Mitú - Pucará)}

Se encuentra emplazado en dolomías grises oscuras con tamaño de cristal grueso, bituminosas y con cavidades rellenas de doloesparita y calcita. Hacia el este, el manto va desapareciendo y se interdigita con dolomita wackestone gris claro (figura 4). La mineralización consiste en esfalerita y galena; ocurre en forma masiva, diseminada, rellenando parcialmente cavidades de disolución $\mathrm{y}$, aisladamente, rellenando fracturas. En el extremo noreste ocurre pirita diseminada y óxidos de hierro. En algunos sectores del manto se observa material brechado conteniendo esfalerita como cemento.

\subsubsection{Mantos intermedio}

Es uno de los mantos más importantes y se emplaza en la dolomía wackestone gris porosa, contienen más evidencia de presencia de evaporitas y dolomías porosas que probablemente sirvieron como un reservorio lateral para hidrocarburos y gases de azufre reducido. El mineral principal es la esfalerita y ocurre diseminada dentro de estructuras bandeadas y en menor cantidad rellenando cavidades. Se observan diseminaciones esporádicas de galena; la pirita se encuentra diseminada al techo y está asociada con bitumen (figura 5).

\subsubsection{Manto resurgidora}

Aflora en superficie de por lo menos $2 \mathrm{~km}$ y con sondajes se identificó un total aproximado de $6 \mathrm{~km}$ de longitud, con potencias que alcanzan hasta los 18 metros, este manto tiene un promedio total de $3 \% \mathrm{Zn}$. Se emplaza en el Miembro Dolomía mudstone beige, en la base son laminares y con estructuras del tipo bird's eyes. La mineralización es pobre y está constituida por esfalerita rellenando pequeñas estructuras bird's eyes, en pequeños lentes entre los planos de estratificación, la galena ocurre en diseminaciones aisladas y pequeñas venillas. La pirita y marcasita son abundantes y están diseminadas en todo 


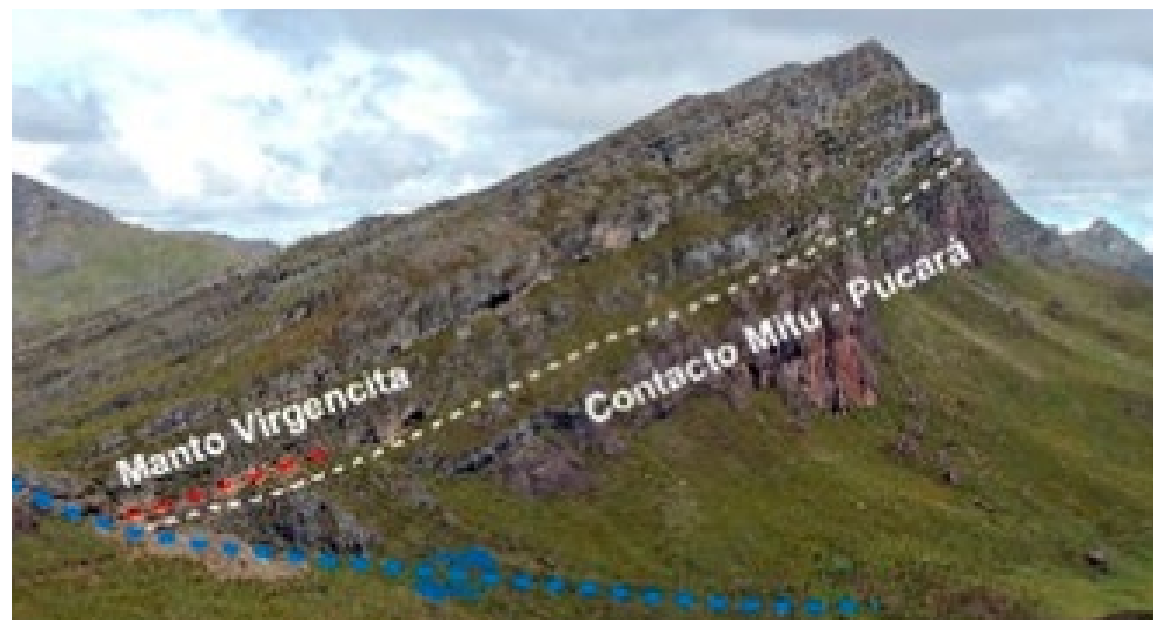

Figura 4. Se observa el manto Virgencita emplazado en las dolomías wackestone grises de la base de la Formación Chambará.

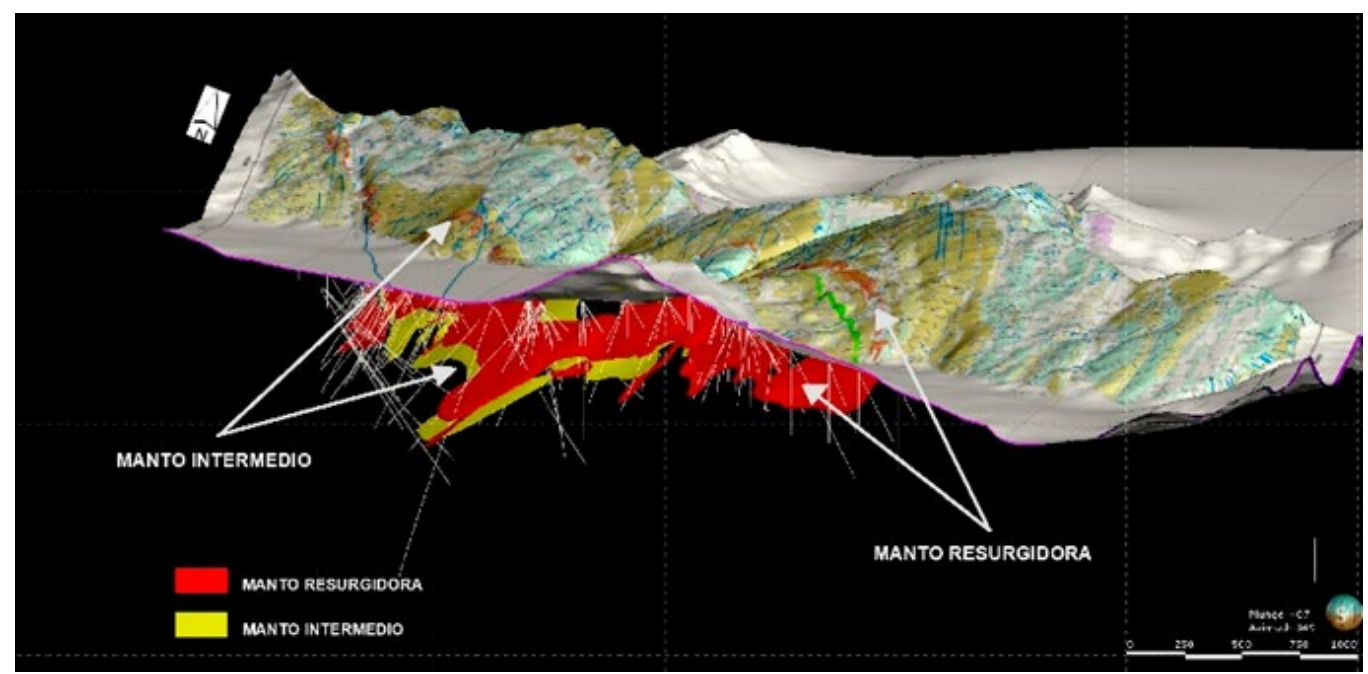

Figura 5. Vista 3d donde se puede observar el manto Resurgidora y manto Intermedio.

el manto. La esfalerita y calcita rellenan la porosidad intercristalina y cavidades, mientras que la baritina aparece principalmente en venillas cortas transversales y paralelas a la estratificación (figura 5).

\subsection{Control litológico}

La mineralización de tipo 'manto' se encuentra emplazadas en rocas de la Formación Chambará en 5 posiciones estratigráficas (figura 3). En base al cartografiado y estudios de columnas estratigráficas detalladas se pudo determinar las secuencias litológicas que controlan la mineralización (figura 6).

\subsubsection{Dolomía wackestone gris inferior}

Tiene como base discordante la zona transicional y como techo calizas nodulares con abundante chert. En algunos sectores, se observa dolomías de grano fino, laminares; en otros sectores, las dolomías descansan inmediatamente sobre el Miembro Basal y presentan morfología lenticular y espesores no mayores a 11 metros. Hacia el techo de este miembro destacan dolomías de grano grueso, sacaroideas, con porosidad tipo vuggy, rellenas con óxidos de hierro, laminación cruzada por sectores y bioclastos (bivalvos, equinodermos y pentacrinos). El espesor promedio de esta unidad es 40 metros. Aquí se aloja el manto Virgencita, en parte en el contacto con el Miembro Basal.

\subsubsection{Dolomía wackestone gris inferior 1}

Está constituida de dolomías de grano medio a grueso con moldes de bioclastos (bivalvos, gasterópodos y equinodermos). Varía lateralmente a facies de calizas principalmente packstone a grainstone bioclásticas $\mathrm{y}$ oolíticas. Presenta un espesor variable, desde 15 metros hasta 115 metros. Dentro de este miembro también podemos encontrar mineralización en mantos en forma irregular, asociado a fallas. 


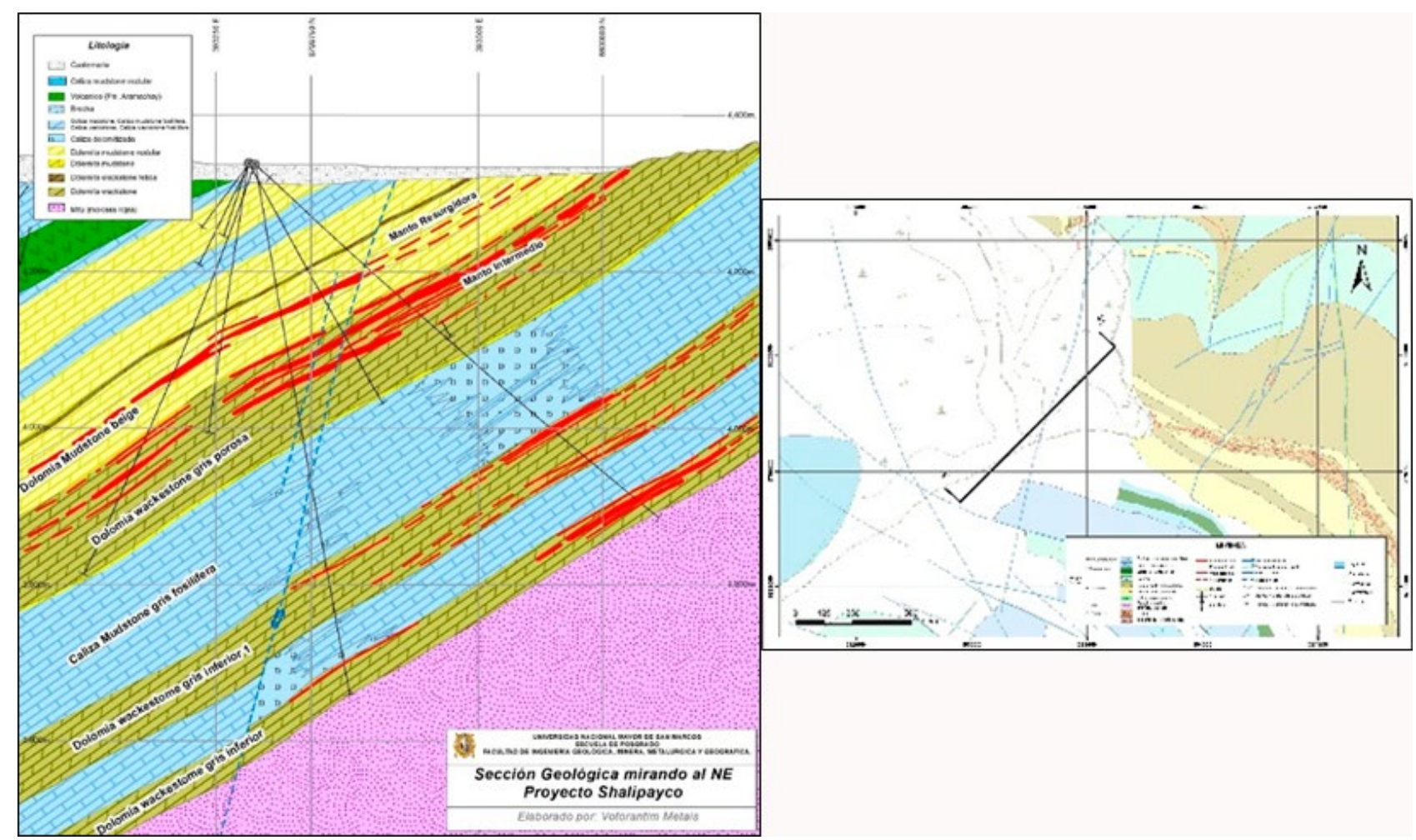

Figura 6. Se muestra una sección mirando al NE donde se puede apreciar los mantos intermedios que se encuentran emplazados en la dolomía wackestone.

\subsubsection{Caliza mudstone gris fosilifera}

Compuesto por una alternancia de calizas lenticulares con abundante chert nodular con calizas en bancos potentes conteniendo fósiles de crinoideos, lamelibranquios y algunas colonias de corales. La textura varía de mudstone a grainstone y ocasionalmente, se intercalan lentes de dolomitas beige de tamaño de cristal medio a grueso, con moldes de bioclastos y chert en nódulos. La potencia promedio es de 100 metros, en la parte intermedia de este miembro, en el sector Pucará estan los mantos Pucará. Las dolomías pasan lateralmente a calizas.

\subsubsection{Dolomía wackestone gris, porosa}

Está compuesto por dolomías beige de tamaño de cristal grueso, por sectores tiene olor fétido. Esta unidad presenta porosidad secundaria tipo "vuggy" rellenada con óxidos de hierro. Los rasgos típicos para su reconocimiento son la ocurrencia de una marcada estratificación cruzada, y la granulometría gruesa; se reporta un espesor mínimo de $40 \mathrm{~m}$. y puede alcanzar espesores hasta de $160 \mathrm{~m}$. Dentro de este miembro se emplazan los mantos Intermedios principales.

\subsubsection{Dolomía mudstone beige}

Litológicamente se compone de dolomías beige de tamaño de cristal fino a medio de una facie sabkha, hacia la base presentan laminación algal e intercalaciones de dolomitas de tamaño de cristal grueso, acá se emplaza el manto Resurgidora, y sobreyacen al Manto Intermedio.

\subsection{Control textural}

Los mantos tienen como roca huésped a las rocas carbonatada con facies evaporíticas, así como las dolomías porosas extensas que probablemente sirvieron como un reservorio lateralmente permeable para hidrocarburos y gases sulfurosos reducidos. Resumimos las texturas:

\subsubsection{Brecha evaporítica}

Brechas que se formaron in situ de la disolución de los minerales evaporíticos (principalmente yeso, anhidrita) o la conversión de estos sulfatos a calcita de grano grueso o dolomita. La brecha sigue la estratificación (Figuras 7A, y 7B).

3.6.2 Transición de una pseudobrecha evaporitica a una brecha evaporítica

En la transición de una brecha evaporítica se refleja el cambio en proporción del carbonato original a minerales de evaporita. Estas zonas pueden ser huéspedes importantes para la mineralización tipo Mississippi Valley debido a la permeabilidad lateral (figura 7C y 7D).

\subsubsection{Brecha de falla}

Las brechas de falla tienen clastos angulares en una matriz de roca disgregada y están asociadas con abundante crackle brecha. A diferencia de otras brechas, los clastos están usualmente en contacto y no son soportadas por matriz de carbonatos de grano grueso. Las fallas y brechas de falla pueden tener alta permeabilidad y pueden ser rutas para la 
"intrusión" de evaporitas y además podrían tener brechas tectónicas de evaporita sobreimpuestas (figura 7E y 7F).

\subsubsection{Pseudobrecha}

Es una textura parecida a una brecha, que puede ser un evento de alteración pre-mena y está asociado a la mineralización. Resulta de la alteración de la roca huésped sin involucrar colapso por disolución o rotación y movimiento de clastos. Un importante control en la distribución lateral de los mantos es la variación lateral de la cantidad de yeso que estaba originalmente presente en los sedimentos de carbonato (figuras $7 \mathrm{G}$ y $7 \mathrm{H}$ ).

\subsubsection{Brecha tectónica evaporítica}

Estas comúnmente migran a lo largo de las fallas o de desprendimientos de cabalgamiento y pareciera estar inyectado en una variedad de fracturas y fallas. Son discordantes a la estratificación, en la zona abunda esta textura, que de acuerdo a las interpretaciones se pudo formar durante la migración de las evaporitas a lo largo del contacto de cabalgamiento produciendo la brecha tectónica evaporítica (figuras $8 \mathrm{~A}$ y $8 \mathrm{~B}$ ). Se reconoce porque los clastos son mayormente angulares y rotados usualmente en matriz soportada por carbonatos gruesos (o en algunos casos por barita o celestita que remplazaron el yeso/ anhidrita) o por fragmentos de roca disgregada, esta textura comúnmente ocurren en secuencias ricas de evaporita que han sido tectónicamente disturbadas.

\subsubsection{Dolomías porosas y vuggy}

Es la textura hospedante mas importante para la mineralización y probablemente sirvió como el reservorio hidrocarburo-azufre reducido, para el evento de mena.

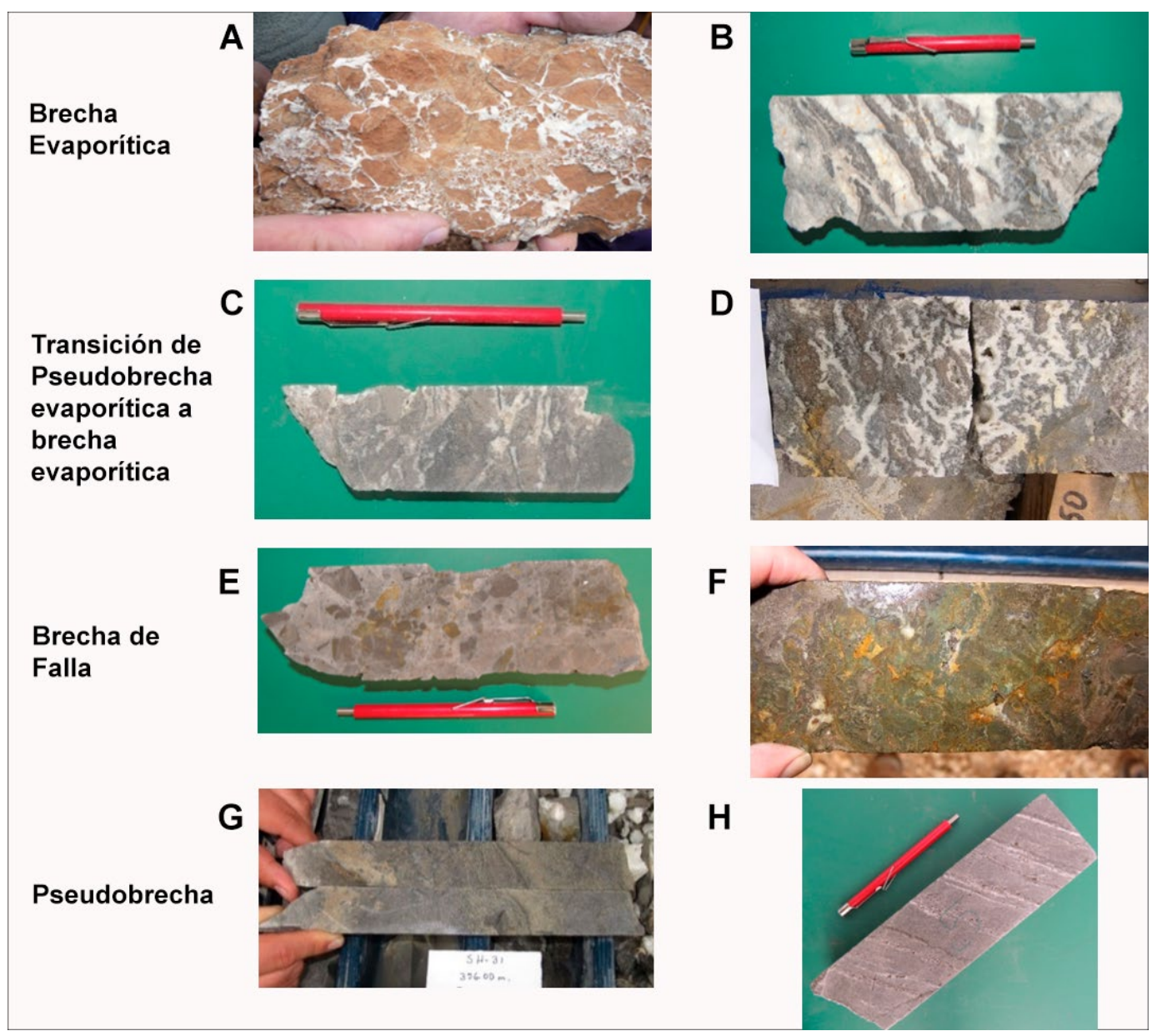

Figura 7. Principales Litologías y Texturas del Proyecto Shalipayco. A) Brecha evaporítica del Manto Resurgidora con clastos angulosos y subredondeados de dolomía mudstone oxidado, con matriz de baritina y calcita. B) Brecha evaporítica, con clastos de dolomía wackestone, y matriz de doloesparita y pirita en parches del Manto Intermedio. C) Transición de una pseudobrecha a brecha evaporitica en dolomía wackestone y doloesparita. D) Transición de una pseudobrecha a brecha evaporítica en dolomía wackestone y doloesparita y esfalerita de color marrón reemplazando algunos clastos de dolomita. Donde refleja el cambio de la proporción de carbonato original a minerales de evaporita. E) Brecha de falla, con clastos angulares de caliza, dolomía y pirita en una matriz de roca disgregada de grano fino. F) Brecha de falla compleja mineralizada con pirita y esfalerita. G) Pseudobrecha del Manto Intermedio en dolomía wackestone gris con esfalerita diseminada, que define la zona permeable lateralmente que probablemente contribuyó a la mineralización del manto. $H$ ) Pseudobrecha evaporítica en dolomía porosa débil, con horizontes de doloesparita. 
La porosidad fue producida probablemente durante la diagénesis de enterramiento y engrosamiento por cabalgamiento andino de la cuenca a través de la disolución de las evaporitas, por conversión de yeso a carbonatos y la disolución de las rocas huéspedes carbonatadas debido a la maduración y producción de ácidos inorgánicos u orgánicos durante la diagénesis, (Leach et al., 2010). La esfalerita comúnmente se encuentra rellenando los poros pero esta también es favorable para un considerable reemplazamiento de carbonatos (figuras $8 \mathrm{C}$ y $8 \mathrm{D}$ ).

\subsubsection{Facies sabkha}

Esta facies es una dolomía de grano fino con evidencia de minerales y evaporita, mayoritariamente la evaporita esta desaparecida debido a que esta pobremente preservada, la facies sabkha que sobreyace al Manto Intermedio podría haber servido como una excelente trampa de hidrocarburo y gas que proporcionaban una gran fuente de azufre reducido y reductantes para el fluido de mena. Las evaporitas pueden ser mezcladas con carbonato de grano fino e intercalarse (es decir, textura cebra y pseudobrecha). Generalmente, muchas rocas de grano fino tienen baja permeabilidad (figuras $8 \mathrm{E}$ y $8 \mathrm{~F}$ ).

\subsubsection{Crackle brecha}

Frecuentemente llamada brecha de mosaico está caracterizada por fragmentos que han sido completamente desplazados. La brecha crackle consiste de abundantes fracturas radiales cerca de zonas de fallas y zonas extensionales asociada con karst y brecha evaporítica. En Shalipayco se observa crackle brecha asociado a mineralización (figuras $8 \mathrm{G}$ y $7 \mathrm{H}$ ).

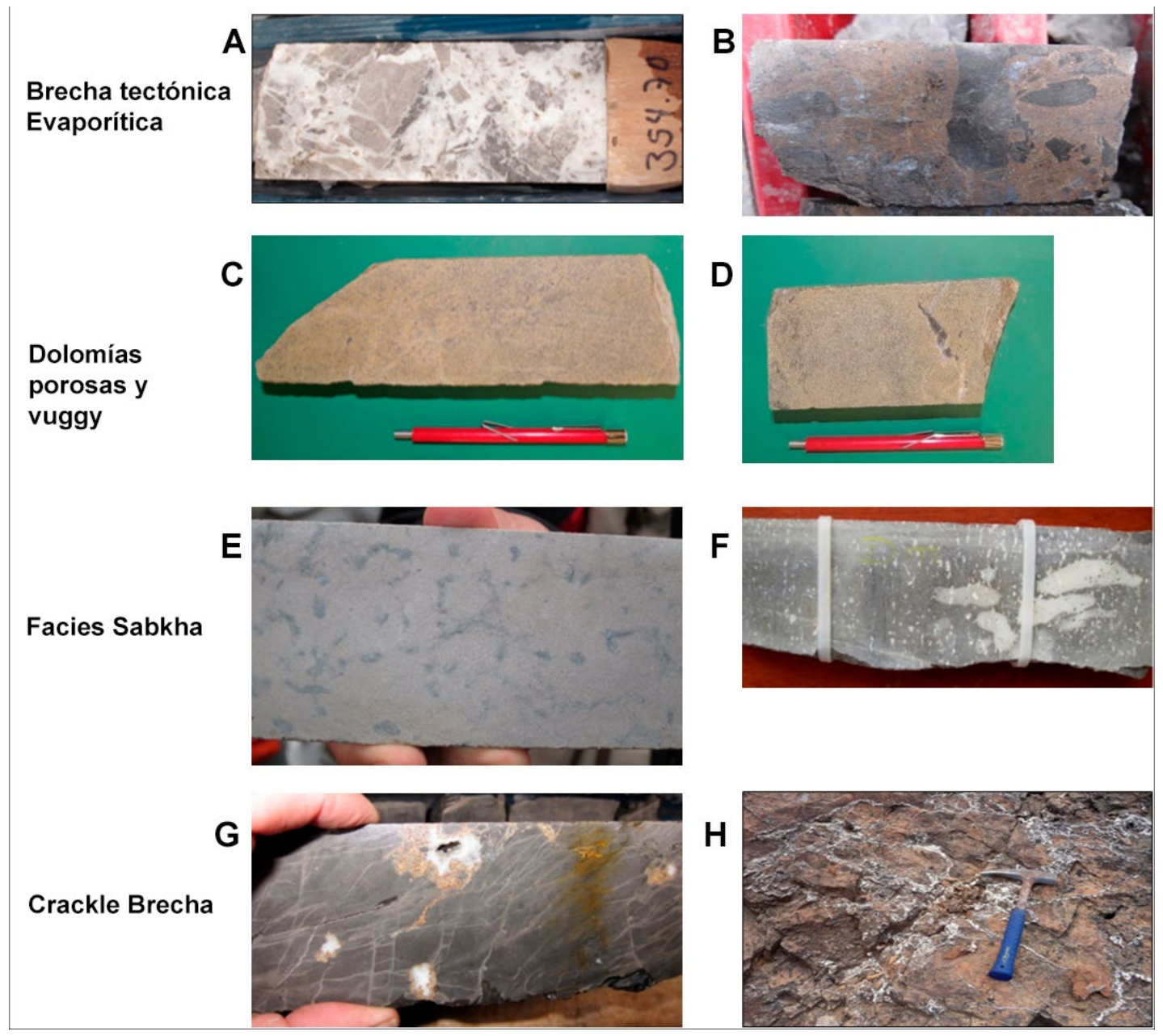

Figura 8. A) Brecha tectónica evaporítica, con clastos angulares y rotados de dolomía wackestone y matriz de doloesparita. B) Brecha tectónica evaporítica, con clastos angulares bituminosos y rotados de dolomía wackestone y matriz de esfalerita. C) Dolomía wackestone del Manto Intermedio, esfalerita reemplazando la roca y rellenando los poros. D) Dolomía wackestone porosa. E) Dolomía de grano fino, con areas diseminadas de yeso ( $¿$ ?) una textura comúnmente presente en las secuencias evaporíticas. F) Dolomía mudstone débilmente porosa de facie sabkha en el Manto Resurgidora que sobreyace al Manto Intermedio, con pseudomorfos de yeso (?) y baritina, pirita diseminada. G) Dolomía mudstone - crackle brecha, nódulos de calcita o ¿yeso? precedente y asociado a esfalerita. H) Se observa crackle brecha en el Manto Resurgidora (dolomía mudstone) que puede ser muy importante para transportar a los fluidos de mena a la roca huésped y además contribuir con la mineralización del depósito. 
Dada las evidencias encontradas en el trabajo de campo, laboratorio y gabinete son 2 los principales controles que guían y controlan la mineralización, la mayor cantidad de mineral está asociado a la litología de roca dolomía, característico en los yacimientos Tipo Mississippi Valley, siendo los principales minerales de la roca la dolomita $\left(\mathrm{CaMg}\left(\mathrm{CO}_{3}\right)_{2}\right)$ y a las texturas de brechas tectónicas, brechas de colapso, porosas "vuggy", cavidades, ritmitas "cebras", cavidades con rellenos de cristales, reemplazamiento de sulfuros. Se cumple que las texturas porosas "vuggy" y brechas evaporíticas tienen una relación directa con la mineralización, esto se ve en los sondajes, el cual corrobora claramente que estas texturas son las que controlan la continuidad lateral de la mineralización. En el horizonte del Manto Resurgidora se encuentra un nivel de caliza dolomítica mudstone de facies sabkha que sobreyace al nivel de dolomía wackestone porosa donde se ubica el manto intermedio, este nivel del manto Resurgidora presenta escasa permeabilidad y no permiten el paso de un fluido lo cual sirvió como roca sello, por lo tanto, se cumple que el nivel de dolomía wackestone pudo ser una excelente trampa de hidrocarburos que proporcionó fuente de azufre reducido y reductores para el fluido de mena. Las relaciones entre la mineralización de los depósitos de tipo Mississippi Valley y las zonas de brecha de intrusión evaporítica (diápiros) tienen importantes implicaciones exploratorias, específicamente la presencia de brecha evaporítica en el campo (especialmente grandes cuerpos discordantes de brecha evaporítica como en San Vicente - Junín), pueden indicar la proximidad de grandes trampas estructurales de hidrocarburos que son locaciones altamente favorables para la mineralización de los depósitos. Es importante conocer la brecha tectónica evaporítica como indicador potencial para la mineralización tipo Mississippi Valley en el Perú, así como en otras locaciones alrededor del mundo. La mineralización Tipo Mississippi Valley, no están típicamente en los cuerpos de brechas pero si en las unidades estratigráficas que fueron reservorios para la reducción de hidrocarburos y azufre.

Realizando una discusión de los resultados, podemos decir que el yacimiento San Vicente es de tipo Mississippi Valley se formó en una facies de barra, y Shalipayco en barra y arrecifes (Dávila \& Valdivia, 2018), en cuanto a la mineralización en San Vicente los fluidos ascendieron por intersección de estructuras N-S y canalizadores hasta alcanzar las rocas reservorio, en Shalipayco los fluidos estan relacionados a fallas N-S y NE, la trampa es más química que sedimentaria. Asimismo, en cuanto a posición estratigráfica de los horizontes productivos se observa que, en SanVicente esta al techo de la Formación Chambara, en Cañón Florida esta casi al piso ,en cambio en Shalipayco esta al medio, lo cual se corrobora con lo encontrado en el presente trabajo donde se concluye que los niveles productivos estan en la zona media de la Formación Chambara. Batista de Oliveira et al., 2019, afirman que el yacimiento Cañón Florida tiene un ensamblaje mineralógico de tipo supergénico dominado por minerales secundarios smithsonita, hemimorfita, cerusita y goethita formados en un clima tropical húmedo durante el mioceno tardio, en cambio la mineralogia observada en Shalipayco es principalmente de minerales primarios conformada por esfalerita, galena y pirita

\section{CONCLUSIONES}

- El control litológico de los mantos principales son las dolomías de grano grueso a medio (wackestone $\mathrm{y}$ packstone) que presentan porosidad tipo "vuggy" y por sectores un olor fétido.

- Las texturas favorables a la mineralización de los principales mantos son las brechas tectónicas evaporíticas, brechas evaporíticas, crackel brecha, pseudobrechas evaporíticas, y dolomías porosas donde las cavidades de disolución están rellenadas por dolomita cristalizada y/o sulfuros de $\mathrm{Zn}$ y $\mathrm{Pb}$. En Shalipayco, las brechas de evaporitas al parecer han contribuido a la permeabilidad lateral de los mantos, especialmente en el caso del Manto Intermedio.

- Es importante la textura de las brecha tectónica evaporítica, ya que pueden generar trampas estructurales de hidrocarburo así como proporcionar una fuente de azufre reducido y puede ser un indicador de áreas favorables para yacimientos tipo Mississippi Valley.

- Basados en la ubicación, observaciones de campo, logueo de rocas carbonáticas asociadas a ambientes evaporíticos, controles generales de formación de mineral, así como las texturas, presencia de brechas evaporíticas, porosidad, dolomitización, presencia presumida de gases o fluidos ricos en azufre reducido, materia orgánica en la roca huésped, son aspectos importantes en yacimientos tipo Mississippi Valley.

\section{REFERENCIAS}

Batista de Oliveira, S., Juliani, C., \& Monteiro, L. V. S. (2019). Mineral characterisation of the non-sulphide $\mathrm{Zn}$ mineralisation of the Florida Canyon deposit, Bongará District, Northern Peru. Applied Earth Science: Transactions of the Institute of Mining and Metallurgy, 128(1), 27-36. https://doi.org/10.1080/25726838.2018.1556033

Dávila, D., \& Valdivia, E. (2018). Estilo y forma de mineralización en el Grupo Pucará MVT: Zn-Pb San Vicente, Shalipayco y Cañón Florida. XIX CONGRESO PERUANO DE GEOLOGÍA. Lima. https://app.ingemmet.gob.pe/ biblioteca/pdf/CPG19-7.pdf

Leach, D. L., Taylor, R. D., Fey, D. L., Diehl, S. F., \& W. Saltus, R. (2010). Mineral Deposit Models for Resource Assessment. In USGS. https://pubs.usgs.gov/sir/2010/5070/a/

MINEM. (2010). Cia. Minera Huarón. Ministerio de Energía y Minas. http://www.minem.gob.pe/minem/archivos/file/ Mineria/REGISTROS/plantas/PLANTAS/P0100085.PDF

MINEM. (2020). Boletín Estadístico Minero. http://www.minem. gob.pe/archivos/BEMDIC2019-z214981zrz0zho.pdf

Rosas, S., \& Fontboté, L. (1989). Evoluvión Sedimentológica del Grupo Pucará (Triásico Superior-Jurásico Inferior) en un perfil SW-NE en el Centro del Perú. Sociedad Geológica Del Perú. https://www.sgp.org.pe/category/ bibliovirtual/?result $=6777$ 
Rev. Inst. INVEStig. FAC. minAs metal. CIENC. GeOGR. 24(47) 2021

Votorantim Metais. (2010). Proyecto de Exploración Minera

Shalipayco. http://intranet2.minem.gob.pe/web/archivos/ dgaam/inicio/resumen/RE_1979704.PDF 